\title{
Dual-foci detection in photoacoustic computed tomography with coplanar light illumination and acoustic detection: a phantom study
}

\author{
Xiangwei Lin \\ Chengbo Liu \\ Jing Meng \\ Xiaojing Gong \\ Riqiang Lin \\ Mingjian Sun \\ Liang Song
}




\section{Dual-foci detection in photoacoustic computed tomography with coplanar light illumination and acoustic detection: a phantom study}

\author{
Xiangwei Lin, ${ }^{a, \dagger}$ Chengbo Liu, ${ }^{b, \dagger}$ Jing Meng, ${ }^{c, \dagger}$ \\ Xiaojing Gong, ${ }^{\text {b, }}$ Riqiang Lin, ${ }^{b}$ Mingjian Sun, ${ }^{a, *}$ and \\ Liang Song ${ }^{\mathrm{b}, \star}$ \\ aHarbin Institute of Technology, Department of Control Science and \\ Engineering, Harbin, China \\ ${ }^{\mathrm{b} C h i n e s e}$ Academy of Sciences, Shenzhen Institutes of Advanced \\ Technology, Research Laboratory for Biomedical Optics and \\ Molecular Imaging, Shenzhen, China \\ ${ }^{\circ}$ Qufu Normal University, College of Computer Science, Rizhao, \\ China
}

Abstract. A dual-foci transducer with coplanar light illumination and acoustic detection was applied for the first time. It overcame the small directivity angle, low-sensitivity, and large datasets in conventional circular scanning or arraybased photoacoustic computed tomography (PACT). The custom-designed transducer is focused on both the scanning plane with virtual-point detection and the elevation direction for large field of view (FOV) cross-sectional imaging. Moreover, a coplanar light illumination and acoustic detection configuration can provide ring-shaped light irradiation with highly efficient acoustic detection, which in principle has a better adaptability when imaging samples of irregular surfaces. Phantom experiments showed that our PACT system can achieve high resolution $(\sim 0.5 \mathrm{~mm})$, enhanced signal-to-noise ratio (16-dB improvement), and a more complete structure in a greater FOV with an equal number of sampling points compared with the results from a flat aperture transducer. This study provides the proof of concept for the fabrication of a sparse array with the dual-foci property and large aperture size for high-quality, low-cost, and high-speed photoacoustic imaging. () 2018 Society of Photo-Optical Instrumentation Engineers (SPIE) [DOI: 10.1117/1. JBO.23.5.050501]

Keywords: photoacoustic computed tomography; dual-foci transducer; virtual-point detection; coplanar light illumination and acoustic detection.

Paper 180009LR received Jan. 15, 2018; accepted for publication Feb. 21, 2018; published online May 8, 2018.

Photoacoustic computed tomography (PACT) has the unique capability of visualizing optical absorbers with high acoustic spatial resolution inside several-centimeter deep biological

\footnotetext{
*Address all correspondence to: Mingjian Sun, E-mail: sunmingjian @ hit.edu. cn; Liang Song, E-mail: liang.song@ siat.ac.cn

${ }^{\dagger}$ First coauthor.
}

tissues, providing anatomical, functional, and molecular contrasts of living biological objects. ${ }^{1-3}$ In traditional circular PACT, photoacoustic (PA) signals can be sampled by scanning a single-element transducer or circular array, where the transducers are usually densely packed with a flat aperture and relatively small size. In practice, the flat aperture can only provide a limited field of view (FOV) due to its narrow directivity angle, resulting in a large data volume and low imaging speed. Further, the small size of the transducer also limits the detection sensitivity. ${ }^{4-6}$

To decrease the data acquisition (DAQ) events, a sparse ultrasonic array and virtual-point detection have been developed. A sparse two-dimensional (2-D) array detector was presented by Ephrat et al. $^{7}$ for fast PA imaging with fewer elements. Wang et al. $^{8}$ utilized a spherical array containing 192 transducers for imaging epilepsy. However, the transducers still had a flat aperture with a limited directivity angle, and the imaging quality was not satisfied with the sparse sampling data. Hence, the virtual-point detector was proposed to expand the directivity angle. ${ }^{9-11} \mathrm{Li}$ et al. $^{9}$ first applied a negative lens in front of a flat transducer to build the virtual-point detector, but the lens would cause the reflection, attenuation, and reverberation of ultrasonic waves that degraded the imaging quality. Concave and convex transducers were also custom-built by Nie et al. ${ }^{12}$ through the curving of the transducer surface, but the resolution in the elevational direction remained poor due to the transducer being cylindrically focused in only one direction. Except for the laser irradiation pattern, the detection sensitivity was also not high enough because of the smaller aperture size.

In this letter, and for the first time to our knowledge, a dualfoci transducer was applied for circular scanning PACT on the condition of coplanar light illumination and acoustic detection. This single-element transducer was custom-built with a different numerical aperture (NA) in two perpendicular directions. A large NA number foci-direction was used to image the scanning plane with a large acceptance angle, while the other direction could provide a fine cross-section resolution and long focal zone with a relatively low NA for imaging the samples. Additionally, the light pattern in the current PACT was far from ideal as it is either shone from the top or oblique side toward the sample, ${ }^{13,14}$ resulting in a low laser energy utilization efficiency. Hence, the coplanar light illumination and acoustic detection were proposed to enable sufficient optical excitation and a highly efficient collection of PA signals.

The schematic of the PACT system integrating the dual-foci transducer (DF-PACT) is shown in Fig. 1(a) and consists of optical illumination, signal acquisition, and data processing units. The optical unit contains a pulsed Nd:YAG laser source (Vibrant 355 II HE, Opotek, Carlsbad) with an optical parameter oscillator that operates in the 410- to 2500-nm wavelength range and emits $8-\mathrm{ns}$ width laser pulses at $10-\mathrm{Hz}$ pulse repetition frequency. The output laser is coupled to a multimode fiber and this bundle is divided into eight branches at the output end. The laser beam out at each branch is reshaped into a slim rectangle with a plano-convex and cylindrical lens. The eight branches are evenly distributed around the sample, enabling ring-shaped light illumination onto the sample surface after the laser beams travel through the water tank and the translucent quartz bowl (top view in the dashed box). The light transmission efficiencies in each branch after penetrating the lens group, tank

$1083-3668 / 2018 / \$ 25.00$ @ 2018 SPIE 


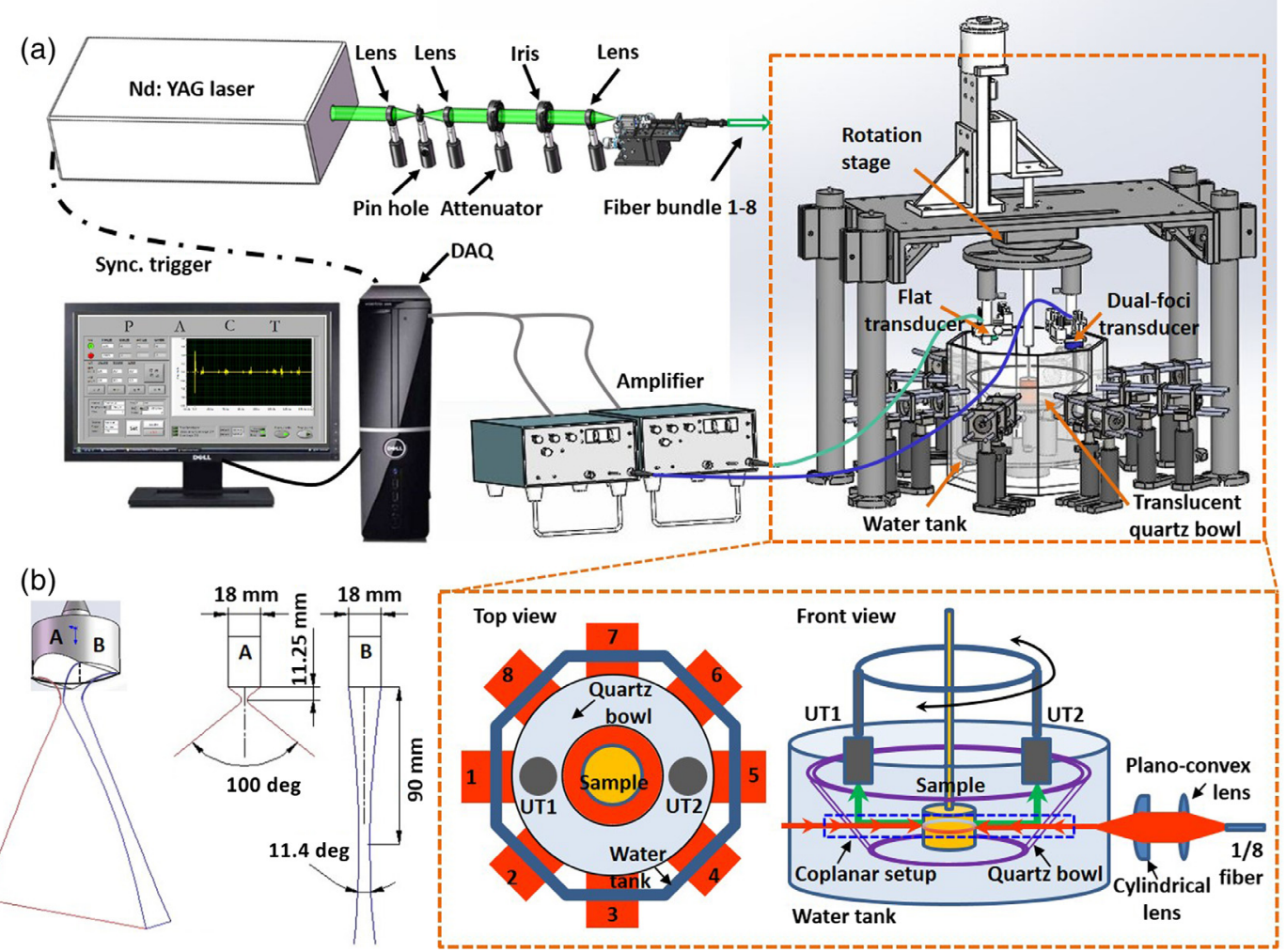

Fig. 1 (a) Schematic of the circular scanning PACT system with a dual-foci and flat aperture transducer and (b) schematic of the dual-foci transducer. UT1, the flat aperture transducer and UT2, the dual-foci transducer.

wall, and quartz bowl separately are $56.05 \%, 64.26 \%, 61.84 \%$, $64.42 \%, 61.24 \%, 61.23 \%, 57.43 \%$, and $60.81 \%$ in the corresponding optical path (as labeled by the numbers in the top view). The average optical fluence to the target surface is $\sim 1.6 \pm 0.2 \mathrm{~mJ} / \mathrm{cm}^{2}$. The PA signals generated from the sample are reflected by the quartz bowl at its inner surface (with a 45 -deg oblique angle), and then the signals are detected by transducers located vertically above the bowl. The ultrasonic reflection efficiency of the quartz bowl was experimentally measured to be $85 \%$. Thus, coplanar light illumination and acoustic detection are achieved (front view in the dashed box).

The virtual-point dual-foci transducer shown in Fig. 1(b) was custom-built by Blatek Inc. It has a 5-MHz center frequency and its detection surface is $18 \mathrm{~mm}$ in diameter. Dual concave crafting was applied at the surface of the dual-foci transducer to achieve focus in two perpendicular directions, each indicated by letters $\mathrm{A}$ and $\mathrm{B}$ in Fig. 1(b). The focal length in direction $\mathrm{A}$ is $11.25 \mathrm{~mm}$ and the directivity angle is $100 \mathrm{deg}(\mathrm{NA}=0.8$ ), while the focal length in direction $\mathrm{B}$ is $90.0 \mathrm{~mm}$ with an 11.4-deg directivity angle $(\mathrm{NA}=0.1$ ). Direction $\mathrm{A}$ is applied in the transverse plane of the DF-PACT system to take full advantage of its large directivity angle for virtual-point detection, and direction $\mathrm{B}$ is used for the elevational focus of the system to obtain a long focal zone $(\sim 5 \mathrm{~cm})$ for imaging the targets in the cross-sectional plane.

In the following experiments, this dual-foci transducer is compared with a common unfocused flat transducer (V310, Olympus, Japan), which also possesses a 5-MHz center frequency but has an $8-\mathrm{mm}$ diameter. These transducers are set geometrically and symmetrically to one another on both sides of the sample and scanned simultaneously along the circular trajectory. Both the sample and the quartz bowl are immersed in the water tank for acoustic coupling. The detected PA signals are amplified with two 39-dB amplifiers (5072 PR, Panametrics) and sent to the DAQ card (ATS330, Alazar, Canada) for realtime sampling. The DAQ card is driven by a personal computer (PC) to transfer the data to the PC for storage and offline processing. The external trigger of the laser is used to synchronize the DAQ card and the mechanical scanning of the transducers. A universal back-projection algorithm ${ }^{15}$ is employed to reconstruct the PA images.

Compared with the previously reported virtual-point detection-based circular scanning PACT, the DF-PACT system in this study should retain much better resolution and sensitivity in the elevational direction benefiting from the elevational focus of the dual-foci transducer. The aperture size and the NA of the dual-foci transducer in the elevational direction lead to a beam diameter of $1.0 \mathrm{~mm}$ at the long focal zone, while the height of the ring-shaped light after transmitting the quartz bowl is $\sim 1.5 \mathrm{~mm}$ with the optical path in our case. Moreover, the light could perpendicularly illuminate to the target surface in the elevational direction, which in principle makes our system robust to any irregular target surface. The elevational focus together with the circular scanning of the transducer enables sufficient coupling of the acoustic detection with the slim ring-shaped optical illumination. Therefore, the elevational resolution theoretically remains at a high level across the entire transverse imaging plane. Also, this slim coplanar configuration has the potential to eliminate out-of-plane artifacts coming from the nearby cross sections, and thus optimal PA signal excitation and detection are achieved and the laser energy is efficiently utilized. 

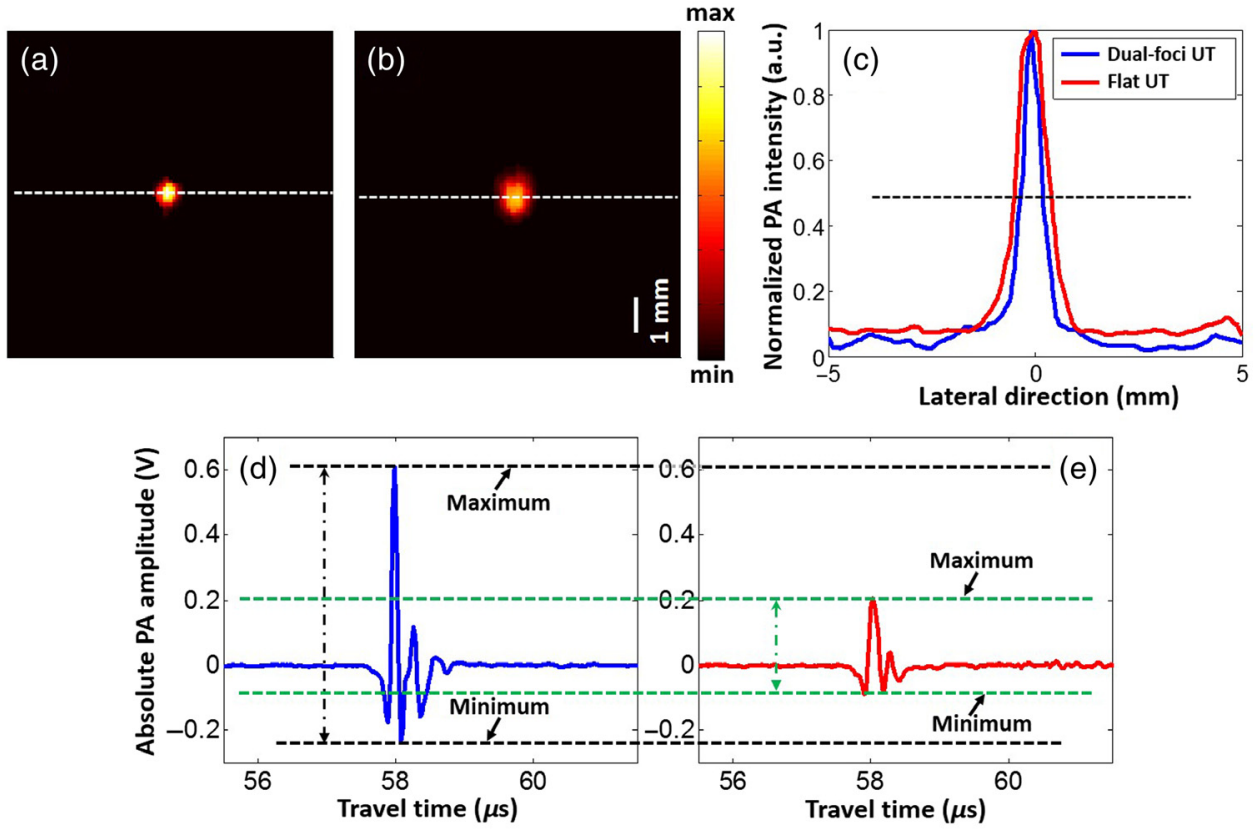

Fig. 2 Reconstructed PA images obtained with (a) the dual-foci transducer and (b) the flat aperture transducer. (c) Normalized PA intensity plots of the dashed centerlines in (a) and (b).

To validate the performance of the DF-PACT system, its spatial resolution and sensitivity in the transverse plane were first evaluated by imaging a point source. A small, point-like PA source with a diameter of $0.3 \mathrm{~mm}$ was placed in the center of the imaging area and the PA signals were detected by the dual-foci transducer and the flat aperture transducer. The scanning radius was $90.0 \mathrm{~mm}$ and the scanning steps were 180 . The corresponding scanning time was $\sim 2$ min. Figures 2(a) and 2(b) show the reconstructed cross-sectional images obtained with the dual-foci transducer and the flat transducer, respectively. It is apparent that the size of the reconstructed point source is smaller with the dual-foci transducer than its counterpart, indicating the higher imaging resolution of the dual-foci transducer. Moreover, Fig. 2(c) shows the normalized PA intensity plots for the two dashed centerlines in Figs. 2(a) and 2(b). The full width at the half maximum of the two plots is separately calculated to be $0.46 \pm 0.02 \mathrm{~mm}$ and $0.98 \pm 0.04 \mathrm{~mm}$ for the dual-foci transducer and the flat transducer, respectively, quantitatively indicating that the dual-foci transducer can reconstruct the target with higher resolution and imaging accuracy (true size of the target is $0.3 \mathrm{~mm}$ ).

The higher detection sensitivity of the dual-foci transducer is further shown in Figs. 2(d) and 2(e), in which the A-line plots corresponding to the raw PA signal obtained with the dual-foci transducer and the flat transducer are depicted, respectively. The two plots were both selected at a random scanning step, as the PA signal varies little during each step of the circular scanning process. Here, the signal-to-noise ratio (SNR) of the two plots was used to evaluate the detection sensitivity of the deep PA signals, which is calculated as the ratio of the PA signal mean value to the background noise standard deviation. The SNR of the PA signal from the dual-foci transducer is $52 \mathrm{~dB}$, which is much larger than the flat transducer case $(36 \mathrm{~dB})$, indicating the higher sensitivity of the custom-made dual-foci transducer. The above advantage of the dual-foci transducer is presumably due to its large aperture size and its elevational focus. It is worth noting that the dual-foci transducer in this study is the only transducer with an elevational focus that has been used in virtual-point detection-based circular scanning PACT and that its aperture diameter is also larger than all the previously reported transducers. Therefore, it is anticipated that this transducer has the highest detection sensitivity to date.

To further verify the performance of the DF-PACT system on the imaging quality under sparse sampling (i.e., reduced scanning steps) when the virtual-point detection is applied, a steel cylinder covered with a layer of black tape was designated the imaging target, as shown in Fig. 3(a). The black tape was circularly scanned with 180,60 , and 20 steps, and the restored images corresponding to the dual-foci transducer and the flat transducer are shown in Figs. 3(b)-3(d) and Figs. 3(e)-3(g), respectively. The quality of the reconstructed images under the sparse sampling deteriorates severely as the scanning steps decrease from 180 to 20 for the flat transducer. However, for the dual-foci transducer, the image quality remains a preferably complete structure, even under the sparsest sampling. Moreover, the PA images obtained with the dual-foci transducer in Figs. 3(b)-3(d) exhibit a much more uniform circular geometry with a better round shape and higher PA signal intensity, indicating the DF-PACT system can obtain much higher imaging accuracy. This is presumably due to the large directivity angle of the dual-foci detector as the virtual-point detection requires fewer scanning steps to be included for the PA image reconstruction. Compared with 180 scanning steps, the acquired dataset would be decreased by 3 times with 60 steps and 9 times with 20 steps. Thus, the DAQ speed could be accelerated by the same folds with the corresponding scanning steps.

In summary, a DF-PACT system with a dual-foci transducer was developed in this study. Based on the virtual-point detector and large FOV resulting from the strong focus in imaging the cross section, high-resolution and high SNR PA results could be obtained with fewer measurements. Thus, a sparse ultrasonic array with virtual point and large size may be explored in PACT, which will simultaneously decrease the system cost and improve the detection sensitivity. Moreover, the coplanar light illumination 

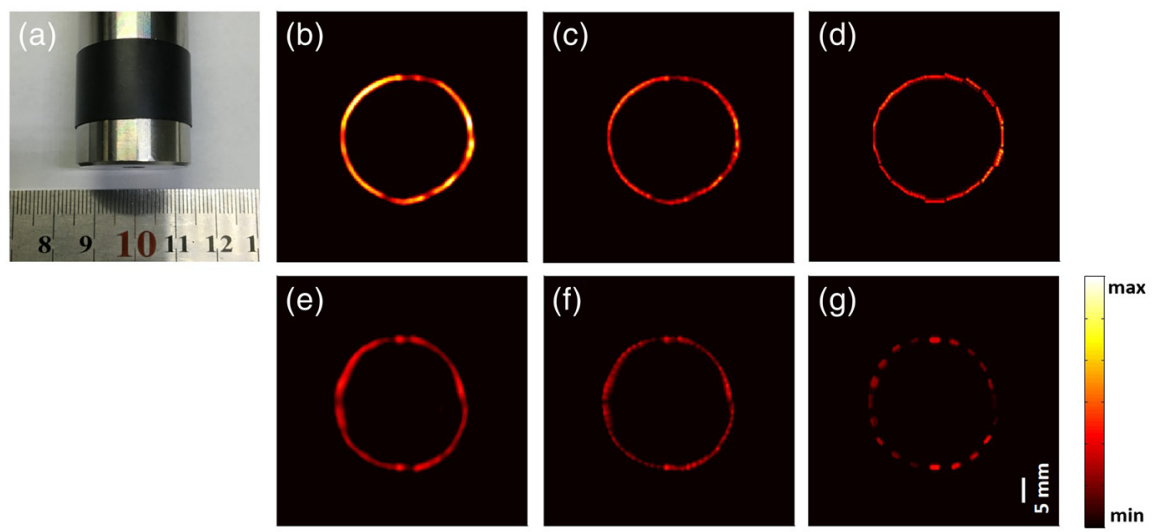

Fig. 3 (a) Photograph of the imaging target. Reconstructed PA images corresponding to 180, 60, and 20 scanning steps obtained with (b)-(d) the dual-foci transducer and (e)-(g) the flat aperture transducer.

and acoustic detection enable highly efficient laser energy utilization and PA signal collection. This study is primarily a proof of concept for the development of a sparse ultrasonic array with dual-foci properties in PACT. To quickly prove this design, we used a handy laser of limited energy output, and only phantom experiments were conducted in this study. In addition, because only one dual-foci transducer was used to sample the PA signals in conjunction with mechanical scanning, in reality, it was very hard to achieve high DAQ speed. However, the advantages of the dual-foci transducer design have been well demonstrated with the phantom experiments carried out in this study. In the future, using the results shown here as the basis, a sparse array transducer with dual-foci property and large element width will be fabricated and integrated into the PACT system to implement real-time 2-D imaging as well as fast 3-D in vivo imaging with higher laser energy. Moreover, the elevational resolution improvement, the merits of imaging targets of irregular surfaces, and the capability of eliminating out-of-plane artifacts will also be further investigated.

\section{Disclosures}

The authors have no relevant financial interests in this letter and no potential conflicts of interest to disclose.

\section{Acknowledgments}

The authors gratefully acknowledge funding from the National Natural Science Foundation of China under Grant Nos. 91739117, 81522024, 81427804, 61405234, 61475182, 61371045, and 61308116; the National Key Basic Research (973) Program of China under Grant Nos. 2014CB744503 and 2015CB755500; the Guangdong Natural Science Foundation under Grant Nos. 2014B050505013 and 2014A030312006; the Shenzhen Science and Technology Innovation under Grant Nos. JCYJ20170413153129570, JCYJ20160531175040976, JCYJ20150521144321005, JCYJ20160608214524052, JCYJ20150401145529015, and GJHS20160331191611536; the Natural Science Foundation of Shandong Province under Grant No. ZR201702220133; and the Key Research and
Development Plan of Shandong Province under Grant Nos. 2015GGX103016 and 2016GGA10094.

\section{References}

1. L. V. Wang et al., "Photoacoustic tomography: in vivo imaging from organelles to organs," Science 335(6075), 1458-1462 (2012).

2. C. Lutzweiler et al., "Optoacoustic imaging and tomography: reconstruction approaches and outstanding challenges in image performance and quantification," Sensors 13(6), 7345-7384 (2013).

3. L. V. Wang et al., "A practical guide to photoacoustic tomography in the life sciences," Nat. Methods 13(8), 627-638 (2016).

4. L. Ding et al., "Real-time model-based inversion in cross-sectional optoacoustic tomography," IEEE Trans. Med. Imaging 35(8), 1883-1891 (2016).

5. C. Zhang et al., "Fast and robust deconvolution-based image reconstruction for photoacoustic tomography in circular geometry: experimental validation," IEEE Photonics J. 2(1), 57-66 (2010).

6. K. P. Köstli et al., "Two-dimensional photoacoustic imaging by use of Fourier-transform image reconstruction and a detector with an anisotropic response," Appl. Opt. 42(10), 1899-1908 (2003).

7. P. Ephrat et al., "Three-dimensional photoacoustic imaging by sparsearray detection and iterative image reconstruction," J. Biomed. Opt. 13(5), 054052 (2008)

8. B. Wang et al., "Photoacoustic tomography system for noninvasive realtime three-dimensional imaging of epilepsy," Biomed. Opt. Express 3(6), 1427-1432 (2012).

9. C. Li et al., "Negative lens concept for photoacoustic tomography," Phys. Rev. E. 78(1), 021901 (2008).

10. X. Yang et al., "Ring-based ultrasonic virtual point detector with applications to photoacoustic tomography," Appl. Phys. Lett. 90(25), 251103 (2007).

11. X. Yang et al., "Monkey brain cortex imaging by photoacoustic tomography," J. Biomed. Opt. 13(4), 044009 (2008).

12. L. Nie et al., "Photoacoustic tomography of monkey brain using virtual point ultrasonic transducers," J. Biomed. Opt. 16(7), 076005 (2011).

13. R. J. Zemp, "Quantitative photoacoustic tomography with multiple optical sources," Appl. Opt. 49(18), 3566-3572 (2010).

14. J. Xia et al., "Whole-body ring-shaped confocal photoacoustic computed tomography of small animals in vivo," J. Biomed. Opt. 17(5), 050506 (2012).

15. M. Xu et al., "Universal back-projection algorithm for photoacoustic computed tomography," Phys. Rev. E. 71(2), 151-155 (2005). 